\title{
ANÁLISE ESPAÇO-TEMPORAL DA EVAPOTRANSPIRAÇÃO DE REFERÊNCIA PARA MINAS GERAIS ${ }^{1}$
}

\author{
Spatial-time analysis of evapotranspiration reference in the Minas Gerais State, Brazil \\ Luis César de Aquino Lemos Filho ${ }^{2}$, Luiz Gonsaga de Carvalho ${ }^{3}$, Adão Wagner Pêgo Evangelista ${ }^{4}$, \\ Luis Marcelo Tavares de Carvalho ${ }^{5}$, Antônio Augusto Aguilar Dantas ${ }^{6}$
}

\begin{abstract}
RESUMO
Objetivou-se com esta pesquisa, analisar a demanda hídrica em Minas Gerais, representada pela evapotranspiração de referência $\left(\mathrm{ET}_{0}\right)$, durante o ano. Os valores de $\mathrm{ET}_{0}$ foram estimados pelo método de Penman-Monteith-FAO a partir de dados diários originados de registros de 42 estações climatológicas do Instituto Nacional de Meteorologia (INMET) referentes a um período de 17 anos (1961 a 1978). No geral, os resultados mostraram que a ET 0 é bastante variável em Minas Gerais, chegando a apresentar valores médios de 914 até valores de $1.677 \mathrm{~mm} \mathrm{ano}^{-1}$. As maiores variações, tanto espaciais como temporais, são registradas no norte do Estado, onde também ocorrem os maiores valores de $\mathrm{ET}_{0}$. O Estado de Minas Gerais apresenta um déficit hídrico anual em aproximadamente $50 \%$ de sua área total. Os meses que apresentaram as maiores e menores demandas hídricas no Estado foram janeiro e junho, respectivamente. Em função da nítida distinção que apresentaram os dados de $\mathrm{ET}_{0}$ geoespacializados nas regiões do Estado de Minas Gerais, o conhecimento do correto valor da $\mathrm{ET}_{0}$ em cada localidade trará benefícios aos produtores no manejo da irrigação.
\end{abstract}

Termos para indexação: Meteorologia, geoprocessamento.

\section{ABSTRACT}

The knowledge of information that expresses the water requirement of the plants is a fundamental issue for the irrigation process. The objective of this research was to analyze the water requirement in Minas Gerais State, Brazil, represented by the evapotranspiration reference $\left(\mathrm{ET}_{0}\right)$, during the year. The $\mathrm{ET}_{0}$ values were estimated through the Penman-Monteith-FAO method starting from daily data originated by the registration of 42 climatological stations of the Instituto Nacional de Meteorologia (INMET) referring to a period of 17 years (1961 to 1978). In general, the results showed that the evapotranspiration reference is plenty variable in Minas Gerais, reaching medium values from 914 to $1.677 \mathrm{~mm}_{\text {year }}{ }^{-1}$. The largest variations, such as spatial and temporal, are registered in the north part of the State, where happen the greatest values of $\mathrm{ET}_{0}$. The Minas Gerais State presents a annual water deficit in approximately $50 \%$ of its total area. The months that presented the greatest and smallest water requirements in the State were January and June, respectively. According to the clear distinction that presented the data of mapping ET in the different regions in the Minas Gerais State, it becames obsvouly that the knowledge of the correct $\mathrm{ET}_{0}$ values in each locality will bring much benefit to farm-producers in the irrigation scheduling.

Index terms: Meteorology, geoprocessing.

\section{(Recebido em 20 de setembro de 2005 e aprovado em 23 de maio de 2006)}

\section{INTRODUÇÃO}

Dada a escassez dos recursos hídricos, o uso da água em áreas irrigadas deve ser o mais racional possível. Assim, determinar o consumo da água das culturas passa a ser um requisito fundamental para o sucesso da irrigação. Para se estimar a evapotranspiração de uma cultura, geralmente é necessário determinar a evapotranpiração de referência $\left(\mathrm{ET}_{0}\right)$, ajustando-a, posteriormente, às condições específicas das culturas e sua fase de desenvolvimento.
Devido à falta de informações climatológicas espacializadas, geralmente, o projetista utiliza informações climatológicas de estações situadas em locais distantes da área de locação do projeto, o que acaba acarretando em erros na estimativa da real demanda hídrica das plantas. Ian \& Wein (1998), citados por Amorim et al. (2003), afirmam que o uso de dados de estações climatológicas próximas pode ser realizado adotando-se a interpolação espacial dos mesmos. O uso dos Sistemas de Informações Geográficas

\footnotetext{
${ }^{1}$ Extraído da dissertação do primeiro autor.

'Engenheiro Agrônomo, MSc., Doutorando em Irrigação e Drenagem - Departamento de Engenharia/DEG- Universidade Federal de Lavras/UFLA - Cx. P. 3037 - 37200-000 - Lavras, MG - lemosfilho@bol.com.br

${ }^{3}$ Engenheiro Agrícola, Professor, Dr., Departamento de Engenharia/DEG - Universidade Federal de Lavras/UFLA - Cx. P. 3037 - $37200-000$ Lavras, MG - Igonsaga@ufla.br

${ }^{4}$ Engenheiro Agrícola, Professor, Dr., Departamento de Engenharia/DEG - Universidade Federal de Lavras/UFLA - Cx. P. 3037 - $37200-000$ Lavras, MG - awpego@ufla.br

${ }^{5}$ Engenheiro Florestal, Professor, Dr., Departamento de Ciências Florestais/DCF- Universidade Federal de Lavras/UFLA - Cx. P. 3037 - $37200-000$ -

Lavras, MG - passarinho@ufla.br

${ }^{6}$ Engenheiro Agrícola, Professor, Dr., Departamento de Engenharia/DEG - Universidade Federal de Lavras /UFLA - Cx. P. 3037 - $37200-000$ Lavras, MG - aadantas@ufla.br
} 
(SIG) na agrometeorologia visa solucionar a ausência de informações regionalizadas. Segundo Pellegrino et al. (1998), uma das aplicações principais do SIG em agrometeorologia é a de transformar dados numéricos, obtidos em pontos referenciados geograficamente na superfície, em mapas interpolados, obtendo-se valores estimados para todas as localidades da região representada. Com isso, gera-se uma série de informações confiáveis a respeito do comportamento espacial da variável, sem a necessidade de observação direta.

Hashmi et al. (1995) concluíram que a versatilidade do SIG, ao considerar as variabilidades espacial e temporal dos elementos climáticos, proporciona aos pesquisadores uma poderosa ferramenta para análise espacial. Comentaram, ainda, que a técnica permite abranger, com muita agilidade e precisão, grandes regiões. Vários autores empregaram técnicas de SIG como ferramenta de análise espacial em projetos sujeitos às influências edafoclimatológicas. Autores como Barbosa et al. (2005), Beltrame et al. (1994) e Chung et al. (1997) espacializaram, com o uso de SIG, a $\mathrm{ET}_{0}$ para diversas regiões. Todos os autores concluíram que a metodologia empregada possibilitou a obtenção de valores estimados individualizados, possibilitando uma estimativa mais precisa da demanda por água em cada localidade.

Tendo em vista o que foi exposto, este trabalho foi desenvolvido com o objetivo de analisar, com o uso de SIG, a variação espacial e temporal da evapotranspiração de referência estimada pelo método de Penman-MonteithFAO para o Estado de Minas Gerais.

\section{MATERIAL E MÉTODOS}

O estudo foi feito para o Estado de Minas Gerais, situado entre os paralelos $14^{\circ} 13^{\prime} 57^{\prime \prime}$ e $22^{\circ} 55^{\prime} 22^{\prime \prime}$ de latitude Sul e os meridianos de $39^{\circ} 51^{\prime} 23^{\prime \prime}$ e $51^{\circ} 02^{\prime} 45^{\prime \prime}$ de longitude oeste. Sua posição, vegetação e topografia oferecem condições climáticas diversificadas e excelente potencial hídrico. Segundo a classificação de Köppen, em Minas Gerais podem ser encontrados os seguintes tipos climáticos: Aw, BSw, Cwa e Cwb, significando que podem ser encontradas desde regiões com clima semi-árido até regiões com clima tropical chuvoso com inverno seco.

O banco de dados que foi utilizado no estudo originou-se de registros de estações classificadas como estações climatológicas principais (ECP) pertencentes à rede nacional de observações meteorológicas de superfície do Instituto Nacional de Meteorologia (INMET). São dados diários, referentes a um período de 17 anos, que vai de 1961 a 1978. As estações selecionadas para o estudo foram aquelas que apresentaram séries históricas mínimas de 5 anos para todos os dados necessários para a estimativa da evapotranspiração de referência $\left(\mathrm{ET}_{0}\right)$ pelo método de Penman-Monteith-FAO descrito por Allen et al. (1998) e Pereira et al. (1997). Para as estações que apresentaram falhas ao longo de suas séries históricas, foi feito o preenchimento das mesmas pelo método da regressão linear simples. Foram utilizadas no trabalho 42 estações climatológicas, das quais, apenas 30 estão localizadas dentro do Estado de Minas Gerais. Para que pudesse ser feita a espacialização das informações, por meio do SIG utilizado no trabalho, foram usados dados de estações climatológicas que se encontram distribuídas nas regiões ou estados circunvizinhos a Minas Gerais. Entre eles estão: Distrito Federal, Goiás, Mato Grosso do Sul, São Paulo, Rio de Janeiro, Espírito Santo e Bahia, totalizando 12 estações fora do limite estadual de Minas Gerais.

No Quadro 1 estão descritas as localizações das diversas estações climatológicas que foram usadas com suas respectivas coordenadas geográficas.

O conjunto de dados foi georreferenciado por meio das latitudes e longitudes em graus, em que, por intermédio de um editor de textos, criou-se um arquivo dBase, contendo as informações X, Y e Z, em que X é a latitude da estação, Y a longitude da estação e Z o valor da variável a ser espacializada. O Datum das estações utilizadas no estudo foi South American Datum 1969 (SAD-69).

O mapa vetorial do contorno do Estado de Minas Gerais, que foi usado como a "máscara", foi obtido por meio de importação de arquivos disponíveis na página da internet do Projeto Geominas (www.geominas.mg.gov.br), de responsabilidade da Secretaria de Estado da Casa Civil do Governo de Minas Gerais. Esses mapas foram gerados pela Companhia de Processamento de Dados do Estado de Minas Gerais (PRODEMG) a partir do mapa geopolítico de Minas Gerais na escala de 1:1.500.000. O sistema de referência é com coordenadas em Albers e o Datum é South American Datum 1969 (SAD-69).

A interpolação foi feita utilizando o Método do Inverso Quadrado da Distância (MIQD), tomando os doze pontos de controle mais próximos (estações meteorológicas com valores medidos para o elemento climático em questão). Isso porque, em testes preliminares, foi o que apresentou os melhores resultados.

Foi feito um balanço hídrico simplificado, através da diferença entre o valor médio total anual da chuva (oferta hídrica) e o valor médio total anual da $\mathrm{ET}_{0}$ (demanda hídrica), desse balanço hídrico originou-se um mapa regionalizado. 
QUADRO 1 - Estações climatológicas principais do INMET em Minas Gerais e estados circunvizinhos.

\begin{tabular}{|c|c|c|c|c|}
\hline Estação (código) & Cidade & Latitude & Longitude & Altitude (m) \\
\hline 83338 & Espinosa, MG & $14^{\circ} 55^{\prime \prime}$ & $42^{\circ} 51^{\prime \prime}$ & 571,22 \\
\hline 83388 & Monte Azul, MG & $15^{\circ} 05^{\prime}$ & $42^{\circ} 45^{\prime}$ & 604,73 \\
\hline 83393 & Pedra Azul, MG & $16^{\circ} 00^{\prime}$ & $41^{\circ} 17^{\prime}$ & 649,91 \\
\hline 83437 & Montes Claros, MG & $16^{\circ} 43^{\prime}$ & $43^{\circ} 52^{\prime}$ & 647,18 \\
\hline 83442 & Araçuaí, MG & $16^{\circ} 52^{\prime}$ & $42^{\circ} 04^{\prime}$ & 285,38 \\
\hline 83479 & Paracatu, MG & $17^{\circ} 13^{\prime}$ & $46^{\circ} 52^{\prime}$ & 712,30 \\
\hline 83481 & João Pinheiro, MG & $17^{\circ} 42^{\prime}$ & $46^{\circ} 10^{\prime}$ & 761,36 \\
\hline 83488 & Itamarandiba, MG & $17^{\circ} 51$ & $42^{\circ} 51^{\prime}$ & $1.097,80$ \\
\hline 83492 & Teófilo Otoni, MG & $17^{\circ} 51^{\prime}$ & $41^{\circ} 31^{\prime}$ & 357,38 \\
\hline 83514 & Capinópolis, MG & $18^{\circ} 41^{\prime}$ & $49^{\circ} 34^{\prime}$ & 621,54 \\
\hline 83531 & Patos de Minas, MG & $18^{\circ} 36^{\prime}$ & $46^{\circ} 31^{\prime}$ & 944,16 \\
\hline 83538 & Diamantina, $\mathrm{MG}$ & $18^{\circ} 15^{\prime}$ & $43^{\circ} 36^{\prime}$ & $1.296,92$ \\
\hline 83543 & Gov. Valadares, MG & $18^{\circ} 51^{\prime}$ & $41^{\circ} 56^{\prime}$ & 278,45 \\
\hline 83570 & Pompeu, MG & $19^{\circ} 13^{\prime}$ & $45^{\circ} 00^{\prime}$ & 691,71 \\
\hline 83577 & Uberaba, MG & $19^{\circ} 45^{\prime}$ & $47^{\circ} 55^{\prime}$ & 743,90 \\
\hline 83579 & Araxá, MG & $19^{\circ} 34^{\prime}$ & $46^{\circ} 56^{\prime}$ & $1.004,67$ \\
\hline 83582 & Bambuí, MG & $20^{\circ} 00^{\prime}$ & $45^{\circ} 59^{\prime}$ & 662,27 \\
\hline 83586 & Sete Lagoas, MG & $19^{\circ} 28^{\prime}$ & $44^{\circ} 15^{\prime}$ & 735,95 \\
\hline 83587 & B. Horizonte, MG & $19^{\circ} 56^{\prime}$ & $43^{\circ} 56^{\prime}$ & 852,28 \\
\hline 83589 & C. Mato Dentro, MG & $19^{\circ} 02^{\prime}$ & $43^{\circ} 26^{\prime}$ & 652,80 \\
\hline 83592 & Caratinga, $\mathrm{MG}$ & $19^{\circ} 48^{\prime}$ & $42^{\circ} 09^{\prime}$ & 611,21 \\
\hline 83595 & Aimorés, MG & $19^{\circ} 29^{\prime}$ & $41^{\circ} 04^{\prime}$ & 83,74 \\
\hline 83632 & Ibirité, MG & $20^{\circ} 01^{\prime}$ & $44^{\circ} 03^{\prime}$ & 815,54 \\
\hline 83639 & Caparão, MG & $20^{\circ} 31^{\prime}$ & $41^{\circ} 52^{\prime}$ & 844,18 \\
\hline 83642 & Viçosa, MG & $20^{\circ} 45^{\prime}$ & $42^{\circ} 51^{\prime}$ & 692,93 \\
\hline 83683 & Machado, MG & $21^{\circ} 40^{\prime}$ & $45^{\circ} 55^{\prime}$ & 877,35 \\
\hline 83687 & Lavras, $\mathrm{MG}$ & $21^{\circ} 14^{\prime}$ & $45^{\circ} 00^{\prime}$ & 920,00 \\
\hline 83689 & Barbacena, MG & $21^{\circ} 15^{\prime}$ & $43^{\circ} 46^{\prime}$ & $1.127,00$ \\
\hline 83692 & Juiz de Fora, MG & $21^{\circ} 46^{\prime}$ & $43^{\circ} 21^{\prime}$ & 941,17 \\
\hline 83736 & São Lourenço, MG & $22^{\circ} 06^{\prime}$ & $45^{\circ} 01^{\prime}$ & 901,32 \\
\hline 83377 & Brasília, DF & $15^{\circ} 47^{\prime}$ & $47^{\circ} 56^{\prime}$ & $1.161,12$ \\
\hline 83379 & Formosa, GO & $15^{\circ} 32^{\prime}$ & $47^{\circ} 20^{\prime}$ & 912,80 \\
\hline 83408 & Carinhanha, BA & $14^{\circ} 10^{\prime}$ & $43^{\circ} 55^{\prime}$ & 440,23 \\
\hline 83446 & Guaratinga, BA & $16^{\circ} 44^{\prime}$ & $39^{\circ} 44^{\prime}$ & 324,86 \\
\hline 83618 & Três Lagoas, MS & $20^{\circ} 47^{\prime}$ & $51^{\circ} 42^{\prime}$ & 313,90 \\
\hline 83630 & Franca, SP & $20^{\circ} 33^{\prime}$ & $47^{\circ} 26^{\prime}$ & $1.027,00$ \\
\hline 83669 & São Simão, SP & $21^{\circ} 29^{\prime}$ & $47^{\circ} 33^{\prime}$ & 618,20 \\
\hline 83714 & Campos do Jordão, SP & $22^{\circ} 44^{\prime \prime}$ & $45^{\circ} 35^{\prime \prime}$ & $1.579,61$ \\
\hline 83726 & São Carlos, SP & $22^{\circ} 01^{\prime}$ & $47^{\circ} 53$ & 856,80 \\
\hline 83646 & C. Itapemirim, ES & $20^{\circ} 51^{\prime}$ & $41^{\circ} 06^{\prime}$ & 78,59 \\
\hline 83695 & Itaperuna, RJ & $21^{\circ} 12^{\prime}$ & $41^{\circ} 53^{\prime}$ & 619,82 \\
\hline 83742 & Vassouras, RJ & $22^{\circ} 20^{\prime}$ & $43^{\circ} 40^{\prime}$ & 438,06 \\
\hline
\end{tabular}

\section{RESULTADOS E DISCUSSÃO}

No gráfico da Figura 1, mostra-se o comportamento dos valores totais mensais médios da evapotranspiração de referência (mm) ao longo do ano para Minas Gerais.
Observa-se, de maneira geral, que, para todas as estações, os valores de $\mathrm{ET}_{0}$ apresentam variações semelhantes ao longo do ano, em que os valores estimados da ET apresentam-se decrescentes de janeiro a junho, passando a ser crescente de junho a dezembro. Os menores valores 
estimados de $\mathrm{ET}_{0}$ ocorreram no período de abril a agosto, tendo os valores mínimos sido no mês de junho. Ainda no gráfico da Figura 1, pode-se observar também que, com algumas exceções, os maiores valores estimados de $\mathrm{ET}_{0}$ para as estações de Minas Gerais foram registrados no mês de janeiro.

No gráfico da Figura 2 encontra-se representado o comportamento dos valores totais mensais médios da precipitação pluvial (mm) ao longo do ano para as estações climatológicas de Minas Gerais. Observa-se que, de forma geral, as variações das precipitações pluviais apresentaram-se semelhantes durante o ano, e que o trimestre mais chuvoso inclui os meses de novembro, dezembro e janeiro, nos quais foram detectados os maiores valores de chuva no estado. O trimestre mais seco é formado pelos meses de junho, julho e agosto, apresentando os menores índices pluviais. Ainda no gráfico da Figura 2 observa-se que, nos meses que apresentaram os maiores índices pluviais, a amplitude dos totais mensais entre as estações foram maiores, existindo desde estações que apresentaram valores de $317 \mathrm{~mm}$ no mês de janeiro até estações que registraram $89 \mathrm{~mm}$ no mesmo mês. Já nos meses de menores índices pluviais, essa amplitude é menor, havendo estações com valores de aproximadamente $31 \mathrm{~mm}$ no mês de agosto até estações com 0 (zero) mm no mesmo mês. Este fato mostra que o Estado de Minas Gerais apresenta grande diversidade climática, possuindo regiões nas quais a precipitação pluvial é abundante até regiões onde esse elemento climático é escasso.
Quando comparados os gráficos das Figuras 1 e 2, pode-se observar que, nos meses em que ocorreram os menores valores de $\mathrm{ET}_{0}$, também ocorreram os menores índices de pluviosidade. Este fato indica que, mesmo que a demanda por água pelas plantas seja menor, a oferta de água da chuva também é pequena, havendo, assim, a necessidade da irrigação para fornecer essa demanda das culturas. Dependendo da região, essa irrigação pode ser de caráter total ou apenas de forma a complementar a oferta da chuva. Isto é melhor visualizado quando observam-se que os menores valores de $\mathrm{ET}_{0}$ não foram inferiores a $40 \mathrm{~mm}$ por mês, enquanto que os menores valores de precipitação pluvial registrados chegaram a zero milímetro $(0 \mathrm{~mm}) \mathrm{em}$ algumas localidades do Estado. Ainda nesta comparação, pode-se observar também que, para a maioria das estações, nos meses em que ocorreram os maiores valores de $\mathrm{ET}_{0}$, também ocorreram os maiores índices de pluviosidade. Índices esses acima dos $100 \mathrm{~mm}$ para quase todas as estações, com exceção das estações da região norte e nordeste do Estado. Isto mostra que mesmo que a oferta de água pela chuva seja grande, a demanda das plantas por água também ocorre numa escala maior. Este fato não descarta o uso da irrigação nessas épocas, principalmente nas regiões norte e nordeste, onde, durante quase todo o período, os valores de $\mathrm{ET}_{0}$ total mensal superam os valores de chuva total mensal. Porém, na maioria das regiões do estado, a necessidade da irrigação pode passar a ser apenas de forma a complementar a demanda hídrica das plantas, visto que apresentam um índice de chuva elevado nessas épocas.

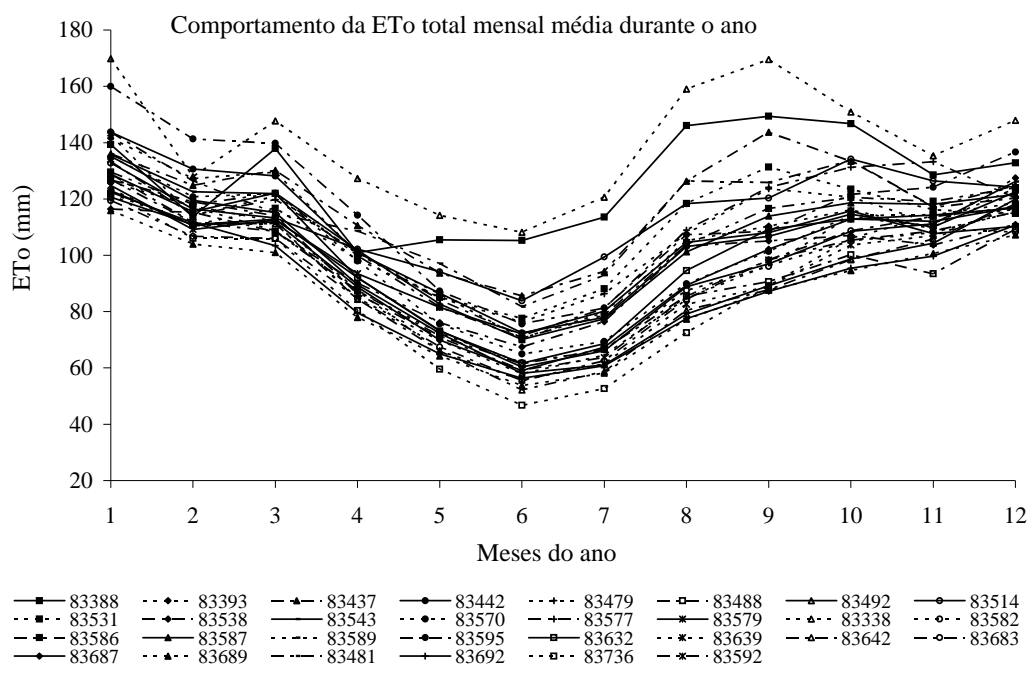

FIGURA 1 - Comportamento dos totais mensais médios da $\mathrm{ET}_{0}(\mathrm{~mm})$ durante o ano. 


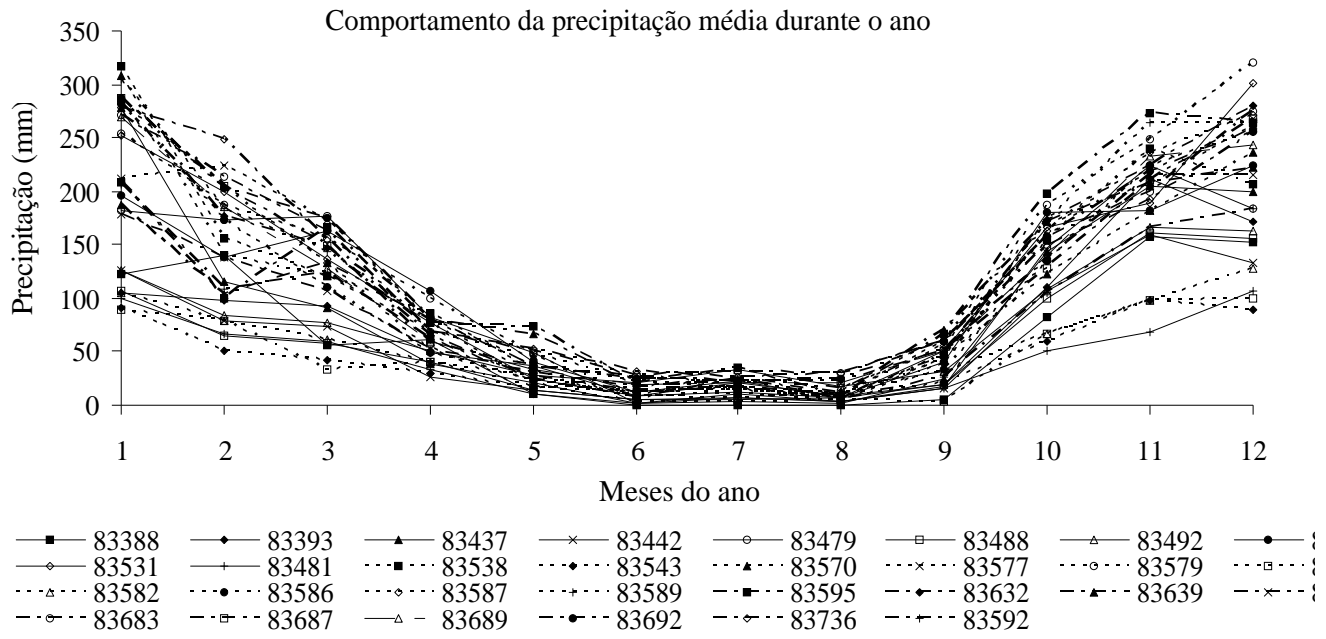

FIGURA 2 - Comportamento dos totais mensais médios da precipitação pluvial (mm) no ano.

Outro motivo para que a irrigação não seja descartada nas épocas de maior precipitação pluvial é o fato das chuvas não ocorrerem com distribuição normal no tempo e no espaço.

Nos mapas das Figuras 3 e 4, tem-se a distribuição espacial da evapotranspiração de referência total anual média $(\mathrm{mm})$ e da precipitação pluvial total anual média $(\mathrm{mm})$, respectivamente, para todo o Estado de Minas Gerais. Podese observar, no mapa da Figura 3, que os menores valores médios de $\mathrm{ET}_{0}$ total anual foram registrados nas regiões Sul, Campo das Vertentes e Zona da Mata, chegando a um valor de $914 \mathrm{~mm}$ ano $^{-1}$ no extremo sul. Observam-se, ainda, no mapa da Figura 3, valores médios crescentes da ET total anual, partindo da região Sul indo em sentido às regiões Noroeste $\mathrm{e}$ Norte, tendo os maiores valores médios da ET total anual sido registrados na região Norte do Estado, chegando ao valor máximo de $1.677 \mathrm{~mm} \mathrm{ano}^{-1}$ no extremo norte. As maiores variações espaciais dos valores de $\mathrm{ET}_{0}$ foram visualizadas nas regiões Norte e Nordeste (Jequitinhonha). Por outro lado, as regiões do Triângulo e Noroeste apresentaram as menores variações espaciais da $\mathrm{ET}_{0}$.

No mapa da Figura 4, observa-se que a precipitação pluvial apresenta comportamento oposto ao da $\mathrm{ET}_{0}$. Observam-se que os menores valores totais anuais médios da chuva foram registrados na região Norte, chegando ao valor mínimo de $512 \mathrm{~mm}^{2} \mathrm{ano}^{-1}$ no extremo norte, enquanto, os maiores valores totais anuais médios de chuva foram registrados na região sul, chegando ao valor máximo de $1.748 \mathrm{~mm} \mathrm{ano}^{-1}$ no extremo sul. A distribuição espacial da chuva em todo o Estado apresentou maior variabilidade quando comparada à distribuição espacial da $\mathrm{ET}_{0}$. Isso se dá pelo fato da chuva ser um elemento meteorológico que apresenta uma alta variabilidade, tanto espacial como temporal. A região Sul foi a que apresentou menor variabilidade espacial da precipitação pluvial, mostrando que se trata de região mais homogênea no que diz respeito a esta variável.

No mapa da Figura 5 é mostrado o balanço hídrico de forma simplificada, envolvendo os mapas das Figuras 3 e 4, a fim de visualizar melhor a oferta e a demanda hídrica nas diversas regiões do Estado. Observa-se, no mapa da Figura 5, que Minas Gerais apresenta déficit hídrico em cerca de $50 \%$ da área total de seu território. As regiões Norte e Nordeste (Jequitinhonha) são aquelas onde a média total anual da evapotranspiração de referência supera a média do total anual da chuva em maior quantidade, apresentando maior déficit de água no ano, havendo localidades nas regiões do extremo norte em que o déficit hídrico chegou a $1.165 \mathrm{~mm}$ ano $^{-1}$.

Esse comportamento, em que a evapotranspiração supera a chuva, é característica peculiar de regiões semiáridas, como é o caso da região Norte e do Jequitinhonha. Nessas regiões de déficit hídrico, o uso da irrigação é fundamental para se obter boa produção agrícola, porém, essa prática deve ser bem manejada para não ocorrer desperdício de água. Por outro lado, nas regiões Sul, Sudeste, Oeste e Central, o valor total anual médio da chuva superou a $\mathrm{ET}_{0}$ anual média, gerando, assim, excesso hídrico nessas regiões. A região onde esse excesso foi maior, chegando a atingir o valor de $833 \mathrm{~mm} \mathrm{ano}^{-1}$, foi no extremo sul. 


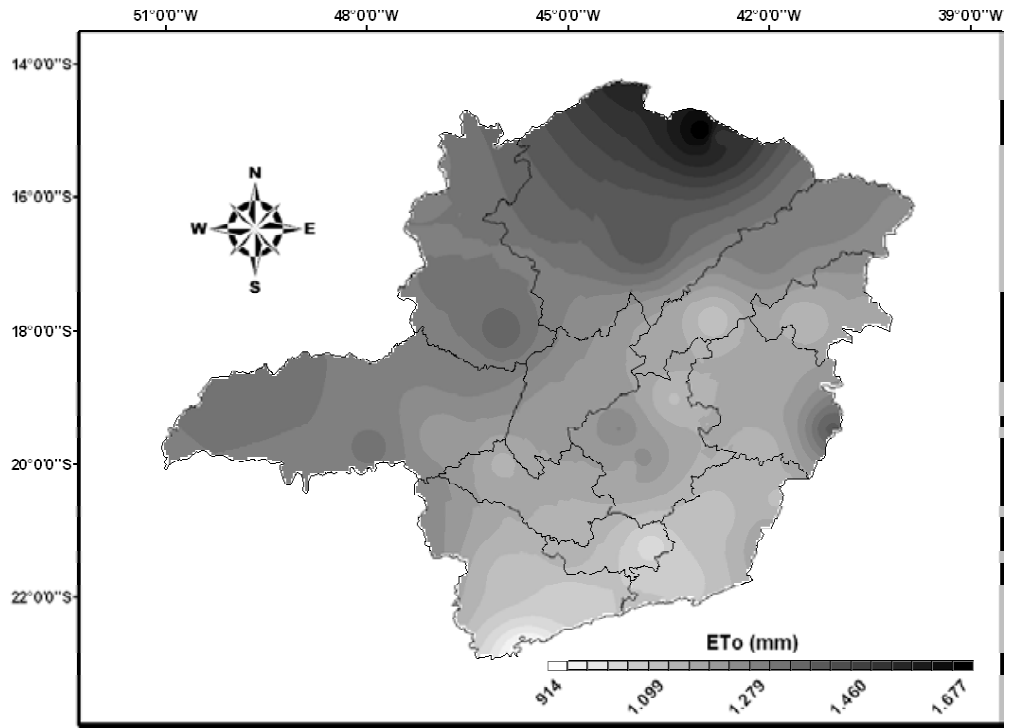

FIGURA 3 - Total anual médio da $\mathrm{ET}_{0}(\mathrm{~mm})$ para o Estado de Minas Gerais.

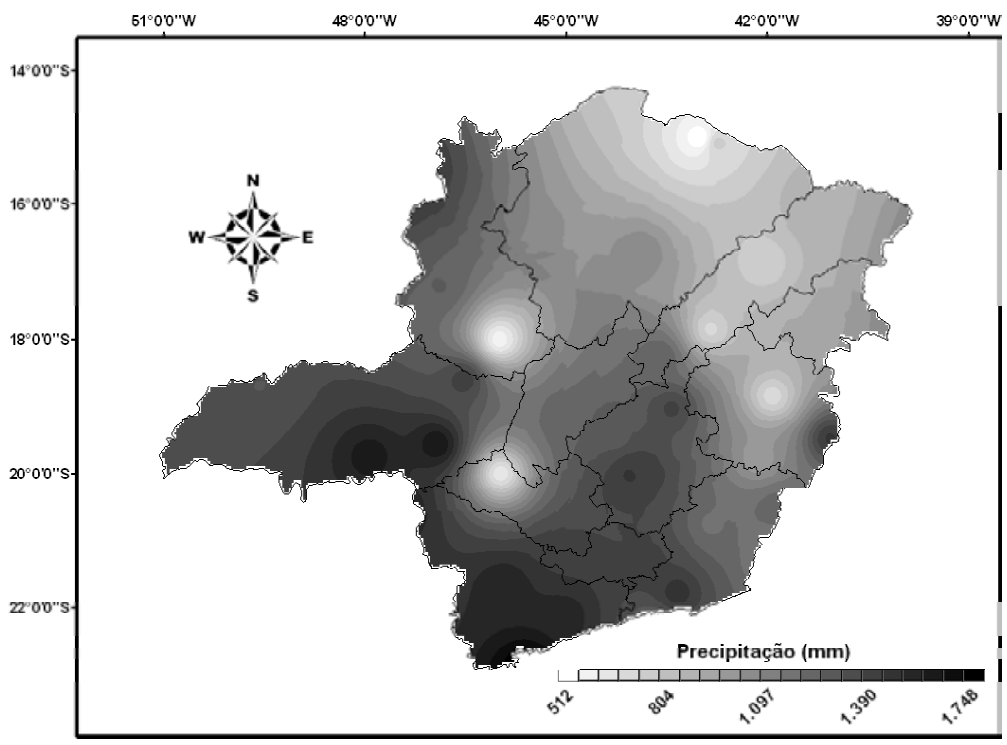

FIGURA 4 - Total anual médio da chuva ( $\mathrm{mm}$ ) para o Estado de Minas Gerais.

Esse fato não descarta o uso da irrigação nessas regiões, porém, mostra a aptidão de algumas localidades para a prática de agricultura sem o uso da irrigação, como é o caso da região Sul, onde grande parte do território é cultivada com a cultura do cafeeiro sem o uso da irrigação. Já na região do Triângulo Mineiro, onde também houve excesso hídrico, porém, em menor escala, a cultura do cafeeiro é realizada, na sua maioria, com o uso da irrigação.

No mapa da Figura 6 é apresentada a distribuição espacial dos coeficientes de variação dos totais anuais da $\mathrm{ET}_{0}$ para todo o Estado. Esse mapa (Figura 6) mostra a 
variação temporal da $\mathrm{ET}_{0}$ estimada para as estações usadas no trabalho. Observam-se que as maiores variações temporais foram registradas nos extremos das regiões Norte e Nordeste (Jequitinhonha), chegando a valores na ordem de $10,29 \%$ de variação no extremo norte do Estado. Nessas regiões podem ocorrer anos com altas demandas hídricas e anos com demandas hídricas reduzidas. Esse comportamento pode ser explicado pelo fato de que regiões de clima árido e semi-árido geralmente apresentam grandes instabilidades climáticas se comparadas a outras regiões, principalmente em relação à precipitação pluvial, que pode influenciar na $\mathrm{ET}_{0}$.
Devido à maior instabilidade da demanda hídrica $\left(\mathrm{ET}_{0}\right)$ nas regiões Norte e Nordeste do estado, os projetistas e usuários da irrigação nessas localidades devem tomar maiores cuidados na estimativa da $\mathrm{ET}_{0}$ na elaboração e manejo de projetos de irrigação.

Já as menores variações temporais foram registradas no noroeste, no oeste, no sul e no sudeste (Zona da mata) de Minas. Essas regiões em que se encontram os menores coeficientes de variação são regiões que, provavelmente, apresentam climas mais estáveis, gerando demandas hídricas semelhantes todos os anos. Isso pode facilitar a prática da irrigação nessas localidades.

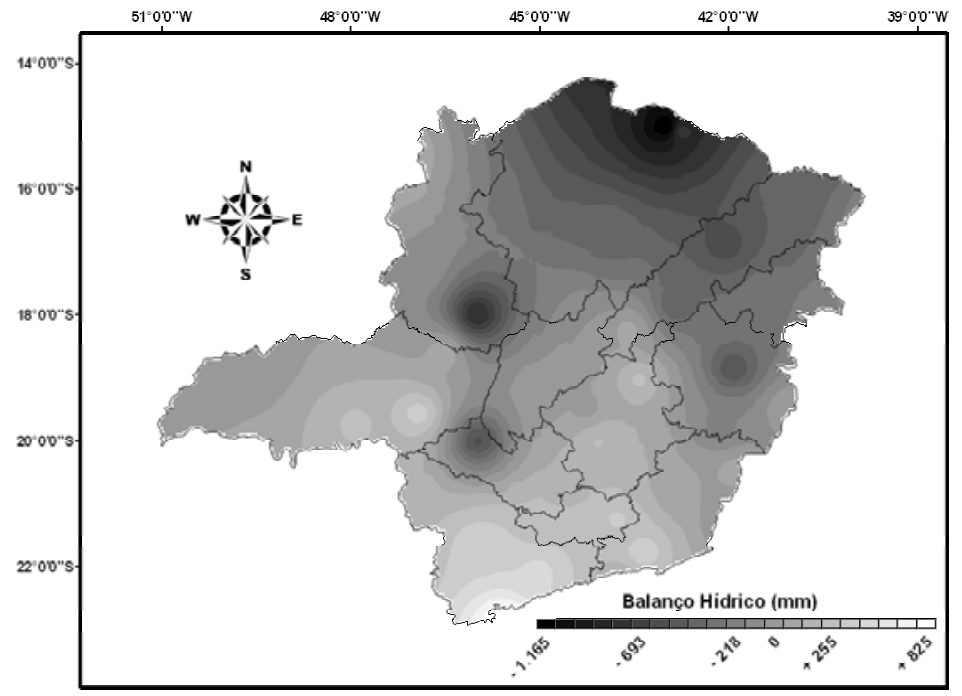

FIGURA 5 - Balanço hídrico simplificado para o Estado de Minas Gerais.

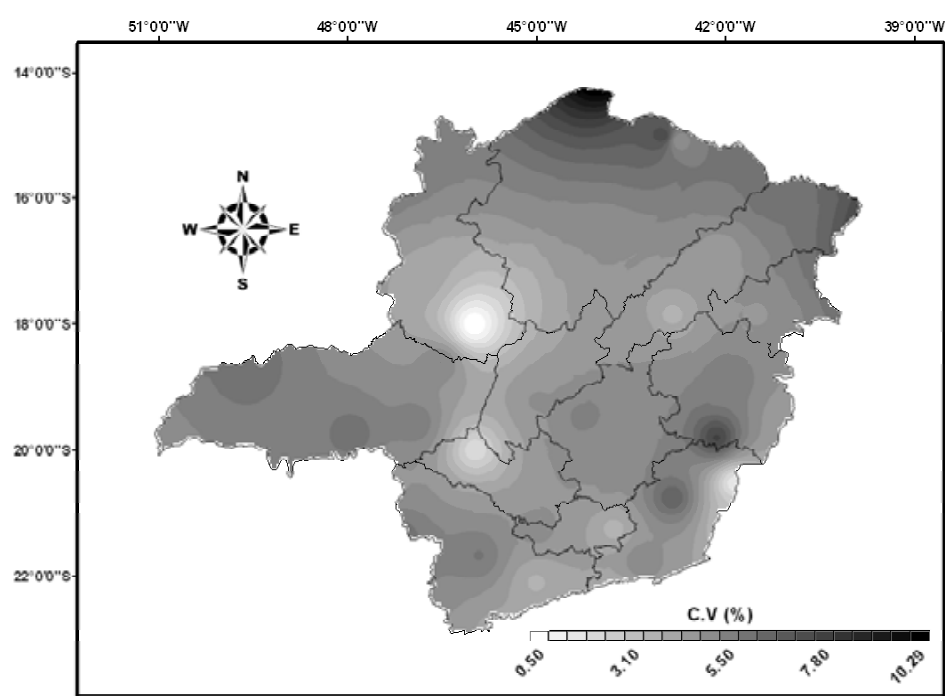

FIGURA 6 - Espacialização dos coeficientes de variação da ET ${ }_{0}$ total anual em Minas Gerais. 


\section{CONCLUSÕES}

As demandas hídricas apresentam grande amplitude de variação no Estado. As maiores variações, tanto espacial como temporal, foram registradas na região Norte. Os menores valores de $\mathrm{ET}_{0}$ ocorrem no mês de junho e os maiores no mês de janeiro. O Estado de Minas Gerais apresenta um déficit hídrico em cerca de $50 \%$ da área total de seu território. Em função da nítida distinção que apresentou os dados de $\mathrm{ET}_{0}$ geoespacializados nas regiões de Minas Gerais, o conhecimento do correto valor da $\mathrm{ET}_{0}$ para cada localidade trará benefícios aos produtores no manejo da irrigação.

\section{REFERÊNCIAS BIBLIOGRÁFICAS}

ALLEN, R. G.; PEREIRA, L. S.; RAES, D.; SMITH, M. Crop evapotranspiration: guidelines for computing crop water requirements. Rome: Food and Agriculture Organization of the United Nations, 1998. 300 p. (FAO Irrigation and Drainage Paper, 56).

AMORIM, R. S. S.; GRIEBELER, N. P.; GONÇALVES, F. A. Comparação de métodos de interpolação para espacialização de dados climáticos. In: CONGRESSO BRASILEIRO DE ENGENHARIA AGRÍCOLA, 2003, Goiânia, GO. Anais... Goiânia: SBEA, 2003. CD-ROM.

BARBOSA, F. C.; TEIXEIRA, A. S.; GONDIM, R. S. Espacialização da evapotranspiração de referência e precipitação efetiva para estimativa das necessidades de irrigação na região do Baixo Jaguaribe-CE. Revista Ciência Agronômica, Fortaleza, v. 36, n. 1, p. 24-33, 2005.

BELTRAME, L. F. S.; LOUZADA, J. A. S.; LANNA, A. E. L. Evapotranspiração potencial do Rio Grande do Sul. Porto Alegre: Instituto de Pesquisas Hidráulicas, 1994. 49 p. (Recursos hídricos, 31).

CHUNG, H. W.; CHOI, J. Y.; BAE, S. J. Calculation of spatial distribution of potential evapotranspiration using GIS. In: ASAE ANNUAL INTERNATIONAL MEETING, 1997, Minneapolis, Minnesota. Paper... Minneapolis: American Society of Agricultural Engineers, 1997. 9 p.

HASHMI, M. A.; GARCIA, L. A.; FONTANE, D. G. Spatial estimation of regional crop evapotranspiration. Transaction of the ASAE, Saint Joseph, v. 38, n. 5, p. 1345-1351, Sept./ Oct. 1995.

PELLEGRINO, G. Q.; PINTO, H. S.; ZULLO JÚNIOR, J.; BRUNINI, O. O uso de sistemas de informações geográficas no mapeamento de informações agrometeorológicas. In: ASSAD, E. D.; SANO, E. E. Sistemas de informações geográficas aplicações na agricultura. Brasília, DF: Embrapa-SPI/Embrapa-CPAC, 1998.

PEREIRA, A. R.; VILLA-NOVA, N. A.; SEDIYAMA, G. C. Evapotranspiração. Piracicaba: FEALQ, 1997. 183 p. 